\title{
Epigenetic control of epithelial-mesenchymal-transition in human cancer (Review)
}

\author{
TOBIAS KIESSLICH ${ }^{1,2}$, MARTIN PICHLER ${ }^{3}$ and DANIEL NEUREITER ${ }^{1}$ \\ ${ }^{1}$ Institute of Pathology; ${ }^{2}$ Department of Internal Medicine I, Paracelsus Medical University/Salzburger \\ Landeskliniken (SALK), Salzburg; ${ }^{3}$ Division of Oncology, Department of Internal Medicine, \\ Medical University of Graz (MUG), Graz, Austria
}

Received August 11, 2012; Accepted September 7, 2012

DOI: $10.3892 / \mathrm{mco} .2012 .28$

\begin{abstract}
Development and tissue homeostasis as well as carcinogenesis share the evolutionary conserved process of epithelial-mesenchymal transition (EMT). EMT enables differentiated epithelial cells to trans-differentiate to a mesenchymal phenotype which is associated with diverse cellular properties including altered morphology, migration and invasion and stemness. In physiological development and tissue homeostasis, EMT exerts beneficial functions for structured tissue formation and maintenance. Under pathological conditions, EMT causes uncontrolled tissue repair and organ fibrosis, as well as the induction of tumor growth, angiogenesis and metastasis in the context of cancer progression. Particularly, the metastatic process is essentially linked to diverse EMT-driven functions which give the mesenchymal differentiated tumor cells the capacity to migrate and form micrometastases in distant organs. Recent analyses of the mechanisms controlling EMT revealed a significant epigenetic regulatory impact reflecting the reversible nature of EMTs. As several approaches of epigenetic therapy are already under clinical evaluation, including inhibitors of DNA methyl transferase and histone deacetylase, targeting the epigenetic regulation of EMT may represent a promising therapeutic option in the future. Therefore, we undertook this review to reassess the current knowledge on the roles of epigenetic control in the regulation of EMT in human cancer. These recent findings are discussed in view of their implications on future diagnostic and therapeutic strategies.
\end{abstract}

\section{Contents}

1. Introduction

2. The current concept of epithelial-mesenchymal transition

Correspondence to: Dr Daniel Neureiter, Institute of Pathology, Paracelsus Medical University/Salzburger Landeskliniken (SALK), Müllner Hauptstrasse 48, 5020 Salzburg, Austria

E-mail: d.neureiter@salk.at

Key words: epithelial-mesenchymal transition, epigenetics, DNA methylation, histone modification, cancer
3. EMT in development and in cancer

4. Epigenetic regulation of EMT

5. Therapeutic options targeting epigenetics and EMT

6. Summary and future directions

\section{Introduction}

Epithelial-mesenchymal transition (EMT) as well the reverse process of mesenchymal-epithelial transition (MET) is essential for development and physiological response to injury (such as wound healing) as well in carcinogenesis (1-3).

Under normal conditions, epithelial cells are linked together as well as to the extracellular matrix environment by different types of intercellular junctions (desmosomes, adherens and tight junctions) enabling tissue maintenance and stability. Epithelial cells can gain the potency to acquire a mesenchymal phenotype to allow for physiological circadian tissue changes but also of tissue loss or damage (4). Interestingly, this process is also associated with an intermediate stem cell phenotype, thus reflecting the highly conserved mechanisms during embryogenesis (5-7).

Over the last few years, the interest in understanding EMT and MET has significantly increased since we further understand the essential role of EMT in cancer progression, particularly during the complex initial processes of tissue invasion and extravasation (8). The regulatory mechanisms of EMT have been intensively investigated and can be described by networks of activating/deactivating signalling pathways. Furthermore, EMT is additionally influenced and regulated by epigenetic mechanisms, such as DNA methylation and histone modifications as well as microRNAs (miRNAs, see below). This epigenetic regulation is particularly important as it accounts for the observed reversibility of EMT-associated processes and the plasticity of (cancer) cells to react upon various external and internal stimuli.

Taken together, these data highlight the complex nature of regulations involved in EMT and provide the basis for development of a new types of drugs specifically targeting EMT in human cancers. In this context, we provide a concise review of the current concepts of EMT in human carcinogenesis and an outlook on therapeutic anti-cancer approaches on the epigenetic level. 


\section{The current concept of epithelial-mesenchymal transition}

According to Kalluri and Weinberg (9) the biological process of EMT is described as follows: i) epithelial cells are tightly integrated in their cellular environment by tight junctions or desmosomes; ii) under the influence of different EMT mediators (such as growth factors or cytokines, discussed in detail below) epithelial cells gain a mesenchymal status, which iii) is associated with different biological properties, particularly the ability to invade and metastasize. EMT refers to a collective series of transcriptional and post-translational events that cause epithelial cells to take on mesenchymal features, thus allowing the cells to separate from the tissue context, lose baso-apical polarity and gain motility (3,10-12) (Fig. 1).

It is of central importance, that EMT processes are reversible so that mesenchymal cells can undergo MET to differentiate back to epithelial phenotypes. This reverse transition plays a key role in the formation of macroscopic metastases in different organs (13).

For experimental approaches it is important to characterize the EMT or MET status of tumor cells to investigate the influence of agonistic or antagonistic acting drugs. Different markers of extracellular (fibronectin, vitronectin) and cellular localization (vimentin, E-cadherin) are suitable to identify the EMT-MET-related differentiation status $(1,12,14,15)$ (Table I). Cellular markers are either cytoplasmic membrane proteins (such as E-cadherin, claudins, occludin, desmoplakin) or cytoplasmic proteins (cytokeratins, vimentin or mucins). In particular, the epithelial phenotype is typically characterized by cytokeratin expression which stabilizes the cytoskeleton of epithelial cells. Additionally, these cytokeratins hierarchically classify the epithelial differentiation status depending on the tissue/organ context as described by Moll et al (16).

One of the fundamental molecular aspects of EMT in converting differentiated epithelial tumor cells into de-differentiated, migratory mesenchymal cells is the repression of epithelial genes, such as E-cadherin, which results in the loss of epithelial cell-cell contacts. For tumor progression, the loss of E-cadherin is a central feature in the early stages of metastasis (17-21), further supporting the involvement of an EMT-like process in metastatic tissue invasion. During EMT, E-cadherin is replaced by $\mathrm{N}$-cadherin, a process referred to as 'cadherin switching' $(22,23)$.

Additionally, intermediary filaments, such as vimentin or smooth muscle cells are used as mesenchymal markers. The intercellular connection status described by the expression pattern of E-cadherin, claudins, occludins or desmoplakin indicate a tissue-integrative epithelial status, whereas the linkage to the extracellular matrix is mediated by glycoproteins, such as fibronectin and vitronectins connecting the extracellular matrix with cellular integrins.

The initiation of the complex process of EMT is triggered by multiple cellular signaling mechanisms including 'classical' developmental pathways, such as Hedgehog, Wnt and Notch, as well as signaling by growth factors including transforming growth factor $\beta$ (TGF $\beta$ ), fibroblast growth factor (FGF), epidermal growth factor (EGF), and, platelet-derived growth factor (PDGF) $(1,11,12)$ (Table II). Additionally, epigenetic mechanisms (discussed below) as well as miRNA-based regulation have been reported $(24,25)$. Insight into the underlying mechanism of the transcriptional regulation of EMT came from the initial identification of the transcription factor, Snail (Snail), as a target of the above-mentioned EMT-promoting signaling pathways, which acts as a direct transcriptional repressor of the E-cadherin gene $(26,27)$. In recent years, additional transcription factors have been identified which repress E-cadherin and mediate the transcriptional initiation of EMT: zinc finger protein Snai2 (Slug) (28), the two-handed zinc finger/homeodomain proteins ZEB1 (SEF1 or ZFHX1A) (29) and ZEB2 (SIP1 or ZFHX1B) (30), the basic helix-loop-helix protein E12/E47 (Tcf3) (31), and Twist (although it is not clear whether the latter directly binds the E-boxes within the E-cadherin promoter).

\section{EMT in development and in cancer}

EMT represents the intersection of different aspects of human development which are sequential rather than parallel processes (2). During the early phase of human development, EMT is involved in morphogenesis and stem cell plasticity required for correct implantation, gastrulation and organogenesis $(32,33)$. In the adult organism, subsequent processes relying on regulated EMT or MET are tissue maintenance allowing for reconstruction or maintenance of tissue, as well as cell homeostasis after inflammatory or degenerative insults. In the case of chronic inflammatory and degenerative diseases, such as organ fibrosis, the EMT/MET system is over-regulated which may lead to organ insufficiency or failure (34). Finally, another cancer-related function of EMT was ascribed to cancer stem cells which are centrally involved in tumor progression, metastasis and recurrence after therapy $(35,36)$.

The involved molecular mechanisms of EMT are summarized in the following paragraphs for i) morphogenesis, ii) chronic diseases and finally for iii) cancer.

EMT in development. In order to enable cells to move to new localities, EMT (and the opposite process, MET) is a central aspect in the developing embryo and has been shown to contribute initially to implantation, gastrulation and subsequently to the development of somites, chondrocytes, cardiac valves, and to nephrogenesis $(37,38)$. The associated molecular steps regulating EMT are highly conserved: as mentioned above, the key players of EMT are the transcription factors, Snail, Twist and ZEB and their important repressor target, E-cadherin. The primary goals of all these EMT-related processes are loss of cell-cell adhesion and polarity and changes in the cell shape, as well as enhanced cell motility and 'invasiveness' during embryonic development for organ maturation as reviewed in detail by Thiery et al (2). Although the upstream regulatory inputs seem heterogeneous at a first glance, some of the master pathways [such as Hedgehog, Wnt, TGF- $\beta$ / bone morphogenetic protein (BMP), FGF and EGF] involved in cancer associated-EMT (Table II) also govern EMT during the different phases of embryogenesis. This emphasizes the biological robustness of these pathways (7) and supports the theory that cancer may be viewed as a deregulated program of development (39). Therefore, it is important to further investigate the role of EMT/MET-related processes in development as this knowledge may be transferred to pathophysiological states, such as chronic disease and carcinogenesis which may subsequently aid in the development of new therapeutic approaches. 
Table I. EMT-related changes in protein expression pattern $(12,14,15)$.

\begin{tabular}{lll}
\hline Upregulation (mesenchymal markers) & Upregulation nuclear localisation & Downregulation (epithelia \\
\hline$\uparrow$ N-cadherin & $\beta$-catenin & $\downarrow$ E-cadherin \\
$\uparrow$ Vimentin & Smad- $2 / 3$ & $\downarrow$ Desmoplakin \\
$\uparrow$ Fibronectin & $\mathrm{NF}-\kappa \mathrm{B}$ & $\downarrow$ Cytokeratin \\
$\uparrow$ MMPs & Snail/2, twist & $\downarrow$ Occludin
\end{tabular}

Bold indicates the most significant changes in marker expression. EMT, epithelial-mesenchymal transition; MMP, matrix metalloproteinase; $\mathrm{NF}-\kappa \mathrm{B}$, nuclear factor $\kappa \mathrm{B}$.

Table II. 'Classical' contextual EMT-inducing pathways in human carcinogenesis.

\begin{tabular}{|c|c|c|}
\hline Pathway & Cancer association & Refs. \\
\hline Bile acids & Hepatobiliary carcinoma cells & $(103,104)$ \\
\hline Bone morphogenetic protein & Tumor cells & $(105)$ \\
\hline $\begin{array}{l}\text { Environmental and social factors } \\
\text { (Nicotine, ultraviolet light) }\end{array}$ & Tumor cells & $(106,107)$ \\
\hline Epidermal growth factor & Tumor cells & $(108)$ \\
\hline Estrogens & Breast and ovarian cancer & $(109,110)$ \\
\hline Fibroblast growth factor & Tumor cells & (111) \\
\hline Hepatocyte growth factor & Liver tumor cells & $(112)$ \\
\hline Hypoxia/autocrine motility factor & Ovarian, pancreatic cancer & $(113-115)$ \\
\hline Integrins & Tumor cells & $(116,117)$ \\
\hline Interleukin-related protein/Interleukin-6 & Tumor cells & $(118,119)$ \\
\hline Notch & Tumor cells & $(105)$ \\
\hline Platelet-derived growth factor & Liver and colon cancer & $(120)$ \\
\hline Prostaglandine (2)/cyclooxygenase 2 & NSCLC cells & $(121,122)$ \\
\hline Scatter factor & Malignant mesothelioma & $(123,124)$ \\
\hline Sonic hedgehog & Tumor cells & $(105)$ \\
\hline Transforming growth factor- $\beta$ & Tumor cells & $(116)$ \\
\hline Vascular endothelial growth factor & Tumor cells & \\
\hline WNT & Tumor cells & $(125)$ \\
\hline
\end{tabular}

EMT, epithelial-mesenchymal transition; NSCLC, non-small cell lung carcinoma.

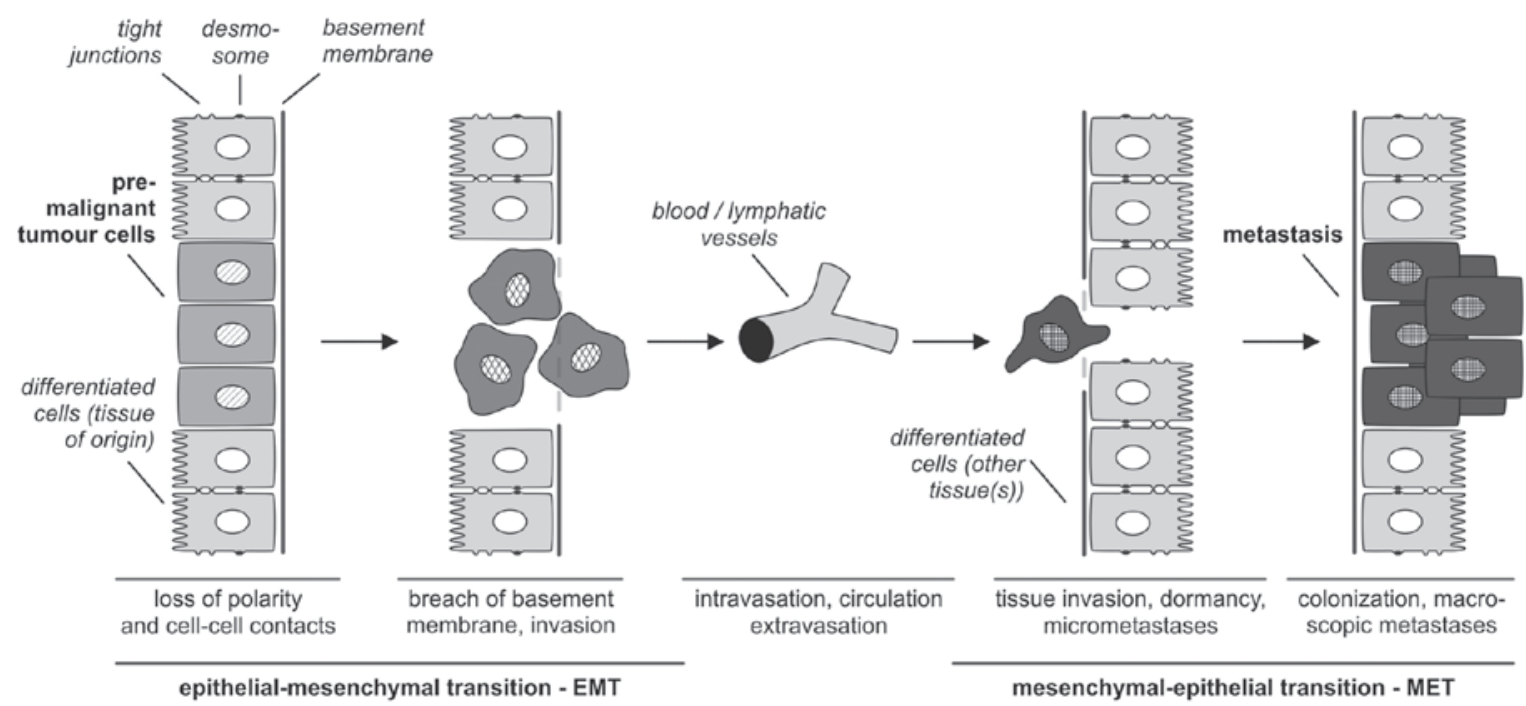

Figure 1. Overview of the initial steps of metastasis: epithelial-mesenchymal transition (EMT) and tissue invasion $(1,12,13)$. 
EMT in chronic diseases. Our knowledge regarding EMT in chronic diseases has increased over the last few years, leading to a new comprehension of chronic diseases. Again, since physiological regeneration and reparation share the same molecular mechanism as EMT/MET in development, it is fascinating to hypothesize that EMT/MET may also play a role in chronic disease caused by over-regulated regeneration and inflammation. This idea is supported by different cellular tracing studies indicating that chronic disease-related interstitial fibrosis is produced by myofibroblasts derived not only from orthotopic fibroblasts, but from epithelial cells via EMT (2). For example, hepatocytes or alveolar epithelial cells differentiate into myofibroblastic cells during carbon tetrachloride (CCl4)-induced liver fibrosis or TGF- $\beta$ treatment, respectively $(40,41)$. Furthermore, endothelial and mesothelial cells have the potency to transdifferentiate into mesenchymal cells relevant for cardiac (42), renal (43) or peritoneal fibrosis (44). As another example, we have previously demonstrated that vascular smooth muscle cells exhibit a reverse molecular epithelial phenotype in human atherosclerosis associated with progressive atherosclerotic lesions (45). The major driving force behind such a type of chronic disease-related fibrosis is TGF- $\beta$ signaling and the Snail cascade which may be inhibited by Smad7 gene transfer (46), as well as a systemic vitamin D analogue (47) or BMP-7 application in vivo (48). This observation may be useful for future therapeutic approaches aiming at protection from progressive organ fibrosis and the associated end stage organ failure.

EMT in cancer. As recently reviewed in depth by Brabletz (8), the de-differentiation processes mediated by EMT are now accepted as a hallmark of cancer. EMT plays a key role in the initial steps of tumor cell dissemination and metastasis. In this context, EMT is related to a current concept of cancer stem cell, i.e. 'migrating cancer stem cells' [as termed by Brabletz et al (5) and Jung et al (49)]. In these models, EMT enables cancer cells to trans-differentiate to mesenchymal cancer cells accompanied by the induction of stem cell-like properties.

Typically, EMT is found locally at the tumor front with a characteristically increased expression of vimentin paralleled by a loss of E-cadherin $(50,51)$. Since EMT is not always obvious in tumor specimens due to the enhanced stromal cellularity at the tumor margin, the relevance of EMT is still under debate (2). Nevertheless, experimental and clinical data on solid tumors, such as breast, colorectal and ovarian carcinoma have revealed that the overexpression of the classical transcription markers, SNAIL1 and SNAIL2, is associated with a worse outcome in terms of relapse or survival (52-54). Additionally, the inhibition of EMT signaling pathways can enhance the efficiency of 'classical' targeted therapy regimes in the experimental setting of hepatic, pancreatic or lung cancer cells $(2,55,56)$. Therefore, detailed topographic analysis of the distribution of EMT markers within the tumor specimen should be carried out for a better prognostic and predictive stratification of cancer patients.

As reviewed by Thiery et al (2) and Brabletz (8), the molecular EMT 'machinery' is synergistically and reciprocally regulated together with other control instances, such as the EMT-inhibiting miRNA-200 and miRNA-34 families influencing differentiation, stemness, proliferation and drug sensitivity. Additionally, the expression of these EMT/MET inducers or inhibitors is under the contextual control of the environment as summarized in Table II.

Taken together, the triggering pathways mentioned in Table II induce Snail gene expression, in turn leading to the repression of E-cadherin by the phosphatidylinositol-3 kinase $(\mathrm{PI} 3 \mathrm{~K}) /$ mitogen-activated protein kinase (MAPK), Smad, RTK, Notch, $\beta$-catenin and glioma-associated oncogene (GLI) signaling cascades, thus further illustrating the complexity of autocrine and paracrine growth factor signaling crosstalk during carcinogenesis and EMT $(2,6,11)$.

For the therapeutic exploitation of these results, different approaches are possible: at the first glance, the EMT transcription factors, TWIST, Snail and the ZEB family, may be targeted to inhibit the EMT process during tumor progression. However, the pharmaceutical potency of available low molecular weight drugs has not been sufficient until now $(2,57)$. RNA interference techniques may represent a promising approach to repress these transcription factors on the mRNA level; however, the in vivo stability and transfer efficiency of this drug technology requires further investigation and optimization (2).

For these reasons, another interesting therapeutic approach may be to target the EMT inducers through small molecular weight inhibitors which have already yielded promising results in an in vitro setting (58). The hierarchical regulatory role of EMT inducers depending on the cancer type should be used as the basis for rational drug selection. Based on the intertwined relationship between EMT processes and cancer stem cells, direct targeting of the latter may also manage the diseaserelated aspects of EMT in cancer. As an example, a promising CSC-targeting drug, salinomycin, was isolated by Gupta et al form a library of 16,000 small molecules (59) and has yielded interesting preclinical results in several tumor entities (60-64).

Additionally, a systematic approach to influence EMT in cancer progression involves modulating the epigenetic regulation of EMT: in the early 80s Jones et al demonstrated that the differentiation status of cultured cells may effectively be influenced by 5 -azacytidine, a hypomethylating agent $(65,66)$. Additionally, it has been shown that the histone-associated chromatin structure, as well as the DNA methylation pattern influence the EMT transcriptional regulation of E-cadherin $(26,27,67,68)$ (described in detail in the following chapter).

\section{Epigenetic regulation of EMT}

Epigenetic regulatory mechanisms refer to a series of stable but reversible modifications not directly affecting the DNA primary sequence but rather rely on dynamic transcriptional programming effects. Such heritable regulations in the pattern of gene expression are mediated by the DNA methylation of $\mathrm{CpG}$ dinucleotides and several post-transcriptional covalent modifications of the $\mathrm{NH}_{2}$ terminal of histone proteins, including acetylation, biotinylation, methylation, phosphorylation and SUMOylation (69). As a general rule, DNA methylation, the di- and trimethylation of $\mathrm{H} 3$ lysine 9 (H3K9) and the trimethylation of H3K27 cause chromatin condensation leading to gene silencing mediated by heterochromatin 1 (HP1) and polycomb group (PcG) proteins (70). Several epigenetic events such as global hypomethylation, specific hypermethylation at $\mathrm{CpG}$ islands $(71,72)$, as well as aberrations in the histone modification landscape ['histone onco-modifications' (73)] have been specifi- 


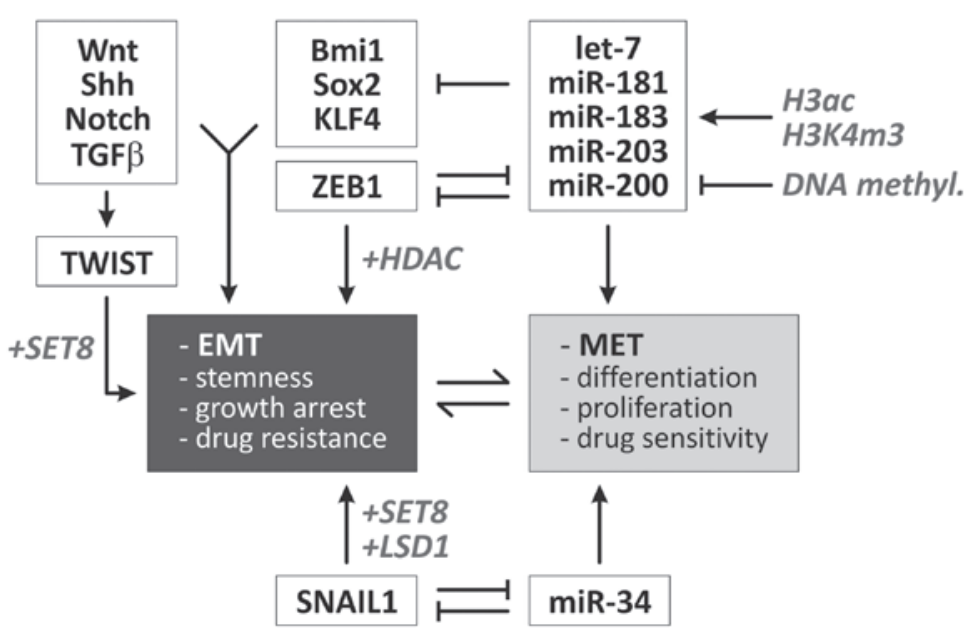

Figure 2. Overview of molecular regulators of EMT. Two negative feedback loops are centrally involved in the dynamic regulation of epithelial vs. mesenchymal cell phenotypes $(1,2)$. Additionally, specific microRNAs and developmental signaling pathways are involved in the regulation of EMT (6). Factors/processes involved in epigenetic control of these pathways/factors are highlighted in gray/italicised (6,16,126-131). Ac, acetylation; EMT, epithelial-mesenchymal transition; H, histone; HDACi, histone deacetylase inhibitor; MET, mesenchymal-epithelial transition; miR, microRNA; m3, trimethylation; DNMTi, DNA methyltransferase inhibitor; Shh, sonic hedgehog.

cally associated with carcinogenesis. This chapter describes the particular findings on how the EMT and EMT-related markers are regulated via epigenetic events. An overview including some of the epigenetic regulatory mechanisms involved in the control of EMT/MET is presented in Fig. 2.

Multiple epigenetic mechanisms have previously been described that act during the EMT program in the repression of epithelial markers and the conversion of epithelial cells into aggressive, invasive tumor cells. In oral carcinoma cells, hypermethylation at the $\mathrm{CDH} 1$ promoter inversely correlates with the expression of E-cadherin and treatment with a demethylating agent (5-azacytidine) causes the re-expression of E-cadherin in cell lines which do not express the SIP1 E-cadherin repressor (74). Similar results have been found in breast tumor cells where $\mathrm{CDH} 1$ promoter hypermethylation rather than mutational inactivation caused the reduced expression of E-cadherin. The expression profile of the cell lines complied with fibroblastic (mesenchymal) morphology and CDH1 promoter hypermethylation (75).

A set of transcription factors has been mechanistically linked to the induction of the EMT program, including Twist, Snail (Snail), Snai2 (Slug) as mediators of the molecular alterations occurring during EMT (76). In several model systems, epigenetic modifications have been shown to contribute to the repressive function of these transcription factors on epithelial genes. As shown by Lin et al (77), Snail recruits the histone demethylase lysine-specific demethylase 1 (LSD1) (KDM1A, AOF2) which removes dimethylation of Lys4 on histone H3 $(\mathrm{H} 2 \mathrm{~K} 4 \mathrm{~m} 2)$ and mediates the transcriptional repression of Snail target genes, such as CDH1. The short-hairpin RNA-mediated depletion of LSD1 results in partial re-expression of epithelial genes associated with increased levels of $\mathrm{H} 3 \mathrm{~K} 4 \mathrm{~m} 2$ at the $\mathrm{CDH} 1$ promoter. These EMT-inducing transcription factors also interact with HDAC1, HDAC2 and the co-repressor mSin3A (74) via their SNAG N-terminal domain as well as polycomb protein repressive complex (PRC2) (77) and cause epigenetic silencing of the CDH1 promoter. Additionally, Yang et al (78) demonstrated that the Twist transcription factor interacts with the monomethyltransferase SET8 which can function both as a repressor (78) or inducer (80) of gene expression. Interestingly, following the interaction of TWIST with SET8, the latter acts as a dual epigenetic modifier on the promoters of $\mathrm{E}$ - and $\mathrm{N}$-cadherin to induce the expression of $\mathrm{N}$-cadherin and the repression of E-cadherin via its H4K20 monomethylation activity (78).

As Snail interacts with several repressor complexes including HDAC, PRC2 and Ajuba-PRMT5 (74,77), Snail causes bivalent histone modifications (e.g., coexistence of $\mathrm{H} 3 \mathrm{~K} 4 \mathrm{~m} 3$ and $\mathrm{H} 3 \mathrm{~K} 27 \mathrm{~m} 3$ ) which render affected genes susceptible to reactivation (81). This is of particular interest as it explains the reversible nature of EMT which, under certain circumstances, can be reversed via MET to generate (e.g., metastasized) cells with epithelial characteristics $(1,13)$.

A large body of evidence demonstrates that the miRNA-200 family and miRNA-205 play an important regulatory role in EMT $(82,83)$. In the context of the epigenetic regulation of EMT, it was found that the CpG island near the miRNA200c and miRNA-141 transcription start is unmethylated in miRNA-expressing tumor/normal cells and is heavily methylated in miRNA-negative and invasive tumor cells. miRNA expression is further facilitated by the enrichment of chromatin-permissive histone modifications (H3 acetylation and H3K4 trimethylation) (84). Likewise, Davalos et al (85) demonstrated that in epithelial cancer cell lines, the 5'-CpG islands of miRNA-200 family members are unmethylated, whereas the hypermethylation-mediated silencing of these miRNAs was found in transformed mesenchymal cells. The reversibility of this methylation state mediates the shift between EMT and MET (85). Similar results were obtained in bladder cancer (86) and breast cancer cell lines (87). It was further shown that ectopic miRNA-200b and -200c expression inhibits ZEB1 translation and disrupts ZEB1-histone deacetylase repressor complexes. This results in increased histone acetylation and E-cadherin expression. Interestingly, the chemo- and radiosensitivity of these breast cancer cells was increased by enhanced p53-mediated apoptotic pathways (88). 


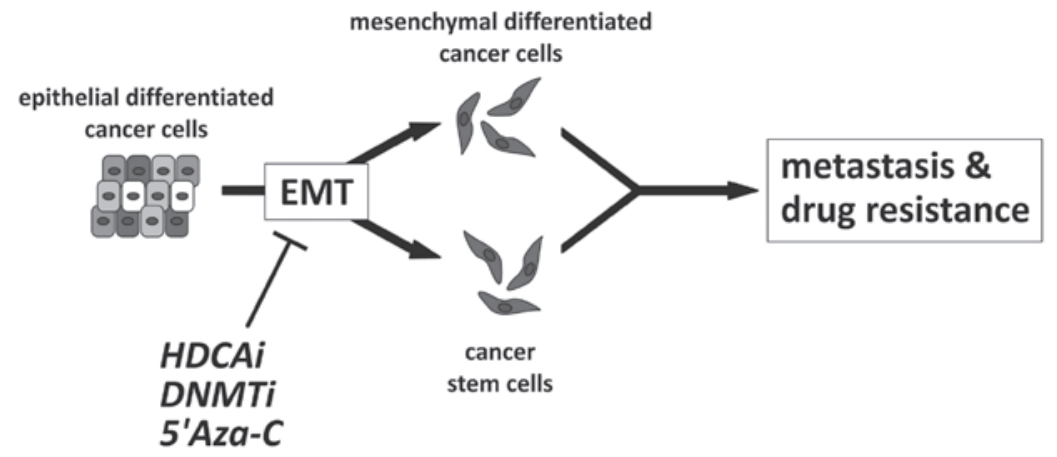

Figure 3. Possible effect of epigenetic cancer therapy targeting EMT. Epithelial-mesenchymal transition contributes to tumor progression by either generating migratory, invasive mesenchymal cancer cells or by the induction of stemness and generation of cancer stem cells, two processes that may involve similar phenomena, i.e., acquisition of stemness and mesenchymal characteristics. Possible epigenetic (classes of) drugs are illustrated to inhibit EMT, including histone deacetylase inhibitor (HDACi), DNA methyltransferase inhibitor (DNMTi) and 5-azacytidine (5-Aza-C) as an example of a demethylating agent $(5,16,96,100,127,128)$.

\section{Therapeutic options targeting epigenetics and EMT}

The overall aim of an epigenetic therapy is to 'renew' the epigenome of the cells by reconstituting the normal expression level of epigenetically misregulated genes (89). Our understanding of the association between modifications of DNA or histones via methylation or acetylation and human diseases has increased over the years, leading to the development of epigenetically functioning drugs, some of which have been approved by the US Food and Drug Administration for the treatment of human cancer (90). As recently reviewed by us, the combination regimen of DNA methyltransferase inhibitors (DNMTi) and histone deacetylase inhibitors (HDACi) yielded promising results in the treatment of myelodysplastic syndrome, a clonal hematological disease (91). Additionally, clinical trials (up to phase IIb) have been performed for other hematological diseases, such as non-Hodgkin's lymphoma (particularly T-cell lymphoma and diffuse large B-cell lymphoma) and acute myeloid leukemia (90). Nevertheless, the clinical application of epigenetic drugs for solid tumors is still in the pilot phase for e.g., non small-cell lung cancer or only used as experimental therapy in advanced, recurrent or refractory malignancies $(90,92)$.

With respect to the molecular effects of these drugs, the detailed mechanisms of epigenetic therapies were primarily focused on their anti-proliferative and pro-apoptotic effects as well as anti-angiogenic potency as supported by many experimental studies in vitro and in vivo $(93,94)$. Of particular interest, recent investigations revealed that acetylation and de-acetylation are centrally integrated in a cellular network of regulations: the 'acetylome' which affects RNA splicing, DNA damage repair, cell cycle control, nuclear transport, actin remodeling, ribosome and chaperone functions $(91,95)$. In our previous studies, we have shown that the cinnamic hydroxamic acid pan-DACi panobinostat (LBH589), a novel potent inhibitor of all HDAC enzymes, influences not only proliferation and apoptosis (96), but also the expression of markers of differentiation and EMT, particularly in an in vivo xenograft model of human hepatoma (97) by upregulation of epithelial markers (cytokeratins) and downregulation of mesenchymal markers (vimentin). Additionally, we demonstrated that the combination of the histone deacetylase inhibitor, SAHA, and the methyltransferase inhibitor, Zebularine, altered the patterns of differentiation in pancreatic cancer models (98). Furthermore, treatment of myelodysplastic syndromes (MDS) and acute myeloid leukemia (AML) with the DNA methylation inhibitor, decitabine (trade name: Vidaza), induced different morphological changes (such as colony forming capacity) and the expression of hematopoietic differentiation markers (99).

These experimental findings are significant since the differentiation status and the associated EMT/MET status of tumor cells is modulated by classical chemotherapy, selecting transitional, stem cell-like tumor cells which are possibly chemotherapy resistant and are responsible for the clinical recurrence as hypothesized by Todaro et al (100). Therefore, the tumor differentiation status should be characterized in detail prior to, during and after tumor treatment in order to obtain 'personalized' predictive, prognostic and therapeutic stratifications. For example, Handra-Luca et al (50) showed that the expression of the 'basic' mesenchymal marker, vimentin, in classical pancreatic ductal adenocarcinoma is associated with a worse outcome of patients using immunohistochemistry on a tissue microarray of 387 patients. Additionally, the same group demonstrated that the loss of E-cadherin protein expression was linked to a worse survival of patients with resectable pancreatic adenocarcinomas (51). As clinicopathological investigations of epigenetic treatment and its impact on EMT/MET in cancer specimen are lacking, to date, only experimental data support the theory that epigenetic treatment of cancer cell lines in vitro and in vivo directly influences the Twist-Snail/ZEB-Ecadherin axis and indirectly influences EMT inducers such as Wnt-TGF 3 -BMP or other classical pathways (as described in chapter 4). Another interesting recent approach was presented by Ivanova et al (101) who investigated the methylation status of different gastric cancer cell lines, revealing that DNA methylation predicts the responsiveness of these cell lines to treatment with cisplatin, a standard chemotherapy for gastric cancer. Additionally, one of the candidate genes, BMP-4, was epigenetically upregulated in cisplatin-resistant gastric cancer cell lines; therefore, the authors speculated that targeting BMP-4 may improve the sensitivity of such cancer cells to chemotherapy (101). Taken together, several tumorigenic properties initiated/driven by EMT such as invasion, metastasis and drug resistance may be targeted by means of epigenetic therapeutic approaches as illustrated in Fig. 3. 


\section{Summary and future directions}

Our understanding of the role of EMT/MET-related processes in different phases of human development, homoeostasis, regeneration and reparation, as well as carcinogenesis has dramatically increased. The central molecular pathways associated with the downstream effects on the most important EMT phenotype, i.e., loss of E-cadherin and vimentin expression have been well described. The direct and indirect inducers of EMT/MET are known and we are beginning to decipher their integrated regulatory crosstalk and feedback mechanisms. As reviewed in this article, the role of epigenetics in EMT is being increasingly strengthened by recent experimental data. Nevertheless, further research is required to fully uncover the whole spectrum of the epigenetic regulation of EMT/MET in human cancer. Based on these insights, novel epigenetic therapies that target the EMT-related processes in tumor progression may become feasible.

With respect to basic cell culture experiments showing the influence of epigenetics on therapy responsiveness or resistance (101), recent data demonstrate that the epigenetic pre-treatment of human cancer cells induces differentiation and, therefore, presents us with a chance to improve the efficiency of classical chemotherapies (102). Therefore, we hypothesize that epigenetic therapy may stabilize the epithelial tumor phenotype or induce MET which may subsequently improve tumor sensitivity to conventional chemotherapy (Fig. 3). However, this hypothesis requires further confirmation in appropriate pre-clinical studies and large prospective clinical trials.

\section{References}

1. Thiery JP and Sleeman JP: Complex networks orchestrate epithelial-mesenchymal transitions. Nat Rev Mol Cell Biol 7: $131-142,2006$

2. Thiery JP, Acloque H, Huang RY and Nieto MA: Epithelialmesenchymal transitions in development and disease. Cell 139: 871-890, 2009

3. Hugo H, Ackland ML, Blick T, Lawrence MG, Clements JA Williams ED and Thompson EW: Epithelial - mesenchymal and mesenchymal - epithelial transitions in carcinoma progression. J Cell Physiol 213: 374-383, 2007.

4. Choi SS and Diehl AM: Epithelial-to-mesenchymal transitions in the liver. Hepatology 50: 2007-2013, 2009.

5. Brabletz T, Jung A, Spaderna S, Hlubek F and Kirchner T: Opinion: migrating cancer stem cells - an integrated concept of malignant tumour progression. Nat Rev Cancer 5: 744-749, 2005

6. Kiesslich T, Berr F, Alinger B, Kemmerling R, Pichler M, Ocker $\mathrm{M}$ and Neureiter D: Current status of therapeutic targeting of developmental signalling pathways in oncology. Curr Pharm Biotechnol 13: 2184-2220, 2012.

7. Kirchner T and Brabletz T: Patterning and nuclear beta-catenin expression in the colonic adenoma-carcinoma sequence. Analogies with embryonic gastrulation. Am J Pathol 157: 1113-1121, 2000.

8. Brabletz T: To differentiate or not - routes towards metastasis. Nat Rev Cancer 12: 425-436, 2012.

9. Kalluri R and Weinberg RA: The basics of epithelial-mesenchymal transition. J Clin Invest 119: 1420-1428, 2009.

10. Guarino M,Rubino B and Ballabio G: The role of epithelial-mesenchymal transition in cancer pathology. Pathology 39: 305-318, 2007.

11. Moustakas A and Heldin $\mathrm{CH}$ : Signaling networks guiding epithelial-mesenchymal transitions during embryogenesis and cancer progression. Cancer Sci 98: 1512-1520, 2007.

12. Voulgari A and Pintzas A: Epithelial-mesenchymal transition in cancer metastasis: mechanisms, markers and strategies to overcome drug resistance in the clinic. Biochim Biophys Acta 1796: 75-90, 2009
13. Scheel C and Weinberg RA: Cancer stem cells and epithelialmesenchymal transition: Concepts and molecular links. Semin Cancer Biol: Apr 23, 2012 (E-pub ahead of print).

14. McConkey DJ, Choi W, Marquis L, et al: Role of epithelial-to-mesenchymal transition (EMT) in drug sensitivity and metastasis in bladder cancer. Cancer Metastasis Rev 28: 335-344, 2009.

15. Ouyang G, Wang Z, Fang X, Liu J and Yang CJ: Molecular signaling of the epithelial to mesenchymal transition in generating and maintaining cancer stem cells. Cell Mol Life Sci 67: 2605-2618, 2010

16. Moll R, Divo M and Langbein L: The human keratins: biology and pathology. Histochem Cell Biol 129: 705-733, 2008.

17. Frixen UH, Behrens J, Sachs M, et al: E-cadherin-mediated cell-cell adhesion prevents invasiveness of human carcinoma cells. J Cell Biol 113: 173-185, 1991.

18. Onder TT, Gupta PB, Mani SA, Yang J, Lander ES and Weinberg RA: Loss of E-cadherin promotes metastasis via multiple downstream transcriptional pathways. Cancer Res 68: 3645-3654, 2008 .

19. Perl AK, Wilgenbus P, Dahl U, Semb H and Christofori G: A causal role for E-cadherin in the transition from adenoma to carcinoma. Nature 392: 190-193, 1998.

20. Schipper JH, Frixen UH, Behrens J, Unger A, Jahnke K and Birchmeier W: E-cadherin expression in squamous cell carcinomas of head and neck: inverse correlation with tumor dedifferentiation and lymph node metastasis. Cancer Res 51: 6328-6337, 1991

21. Umbas R, Isaacs WB, Bringuier PP, et al: Decreased E-cadherin expression is associated with poor prognosis in patients with prostate cancer. Cancer Res 54: 3929-3933, 1994.

22. Cavallaro U, Schaffhauser B and Christofori G: Cadherins and the tumour progression: is it all in a switch? Cancer Lett 176: $123-128,2002$.

23. Maeda M, Johnson KR and Wheelock MJ: Cadherin switching: essential for behavioral but not morphological changes during an epithelium-to-mesenchyme transition. J Cell Sci 118: 873-887, 2005.

24. Gregory PA, Bert AG, Paterson EL, et al: The miR-200 family and miR-205 regulate epithelial to mesenchymal transition by targeting ZEB1 and SIP1. Nat Cell Biol 10: 593-601, 2008.

25. Park SM, Gaur AB, Lengyel E and Peter ME: The miR-200 family determines the epithelial phenotype of cancer cells by targeting the E-cadherin repressors ZEB1 and ZEB2. Genes Dev 22: 894-907, 2008.

26. Batlle E, Sancho E, Franci C, Dominguez D, Monfar M, Baulida J and Garcia DH: The transcription factor snail is a repressor of E-cadherin gene expression in epithelial tumour cells. Nat Cell Biol 2: 84-89, 2000.

27. Cano A, Perez-Moreno MA, Rodrigo I, et al: The transcription factor snail controls epithelial-mesenchymal transitions by repressing E-cadherin expression. Nat Cell Biol 2: 76-83, 2000.

28. Bolos V, Peinado H, Perez-Moreno MA, Fraga MF, Esteller M and Cano A: The transcription factor Slug represses E-cadherin expression and induces epithelial to mesenchymal transitions: a comparison with Snail and E47 repressors. J Cell Sci 116: 499-511, 2003.

29. Grooteclaes ML and Frisch SM: Evidence for a function of $\mathrm{CtBP}$ in epithelial gene regulation and anoikis. Oncogene 19: 3823-3828, 2000

30. Comijn J, Berx G, Vermassen P, et al: The two-handed E box binding zinc finger protein SIP1 downregulates E-cadherin and induces invasion. Mol Cell 7: 1267-1278, 2001.

31. Perez-Moreno MA, Locascio A, Rodrigo I, Dhondt G, Portillo F, Nieto MA and Cano A: A new role for E12/E47 in the repression of E-cadherin expression and epithelial-mesenchymal transitions. J Biol Chem 276: 27424-27431, 2001.

32. Nakaya Y and Sheng G: Epithelial to mesenchymal transition during gastrulation: an embryological view. Dev Growth Differ 50: 755-766, 2008

33. Qin Q, Xu Y, He T, Qin C and Xu J: Normal and disease-related biological functions of Twist1 and underlying molecular mechanisms. Cell Res 22: 90-106, 2012.

34. Piera-Velazquez S, Li Z and Jimenez SA: Role of endothelialmesenchymal transition (EndoMT) in the pathogenesis of fibrotic disorders. Am J Pathol 179: 1074-1080, 2011.

35. Hanahan D and Weinberg RA: Hallmarks of cancer: the next generation. Cell 144: 646-674, 2011

36. Hanahan D and Weinberg RA: The hallmarks of cancer. Cell 100: 57-70, 2000. 
37. Chaffer CL, Thompson EW and Williams ED: Mesenchymal to epithelial transition in development and disease. Cells Tissues Organs 185: 7-19, 2007.

38. Yang $\mathrm{J}$ and Weinberg RA: Epithelial-mesenchymal transition: at the crossroads of development and tumor metastasis. Dev Cell 14: 818-829, 2008

39. Neureiter D, Herold C and Ocker M: Gastrointestinal cancer - only a deregulation of stem cell differentiation? (Review). Int J Mol Med 17: 483-489, 2006

40. Zeisberg M, Yang C, Martino M, Duncan MB, Rieder F, Tanjore $\mathrm{H}$ and Kalluri R: Fibroblasts derive from hepatocytes in liver fibrosis via epithelial to mesenchymal transition. J Biol Chem 282: 23337-23347, 2007.

41. Kim KK, Kugler MC, Wolters PJ, et al: Alveolar epithelial cell mesenchymal transition develops in vivo during pulmonary fibrosis and is regulated by the extracellular matrix. Proc Natl Acad Sci USA 103: 13180-13185, 2006.

42. Zeisberg EM, Tarnavski O, Zeisberg M, et al: Endothelial-tomesenchymal transition contributes to cardiac fibrosis. Nat Med 13: 952-961, 2007.

43. Zeisberg EM, Potenta SE, Sugimoto H,Zeisberg M and Kalluri R: Fibroblasts in kidney fibrosis emerge via endothelial-to-mesenchymal transition. J Am Soc Nephrol 19: 2282-2287, 2008.

44. Yanez-Mo M, Lara-Pezzi E, Selgas R, et al: Peritoneal dialysis and epithelial-to-mesenchymal transition of mesothelial cells. N Engl J Med 348: 403-413, 2003.

45. Stintzing S, Ocker M, Hartner A, Amann K, Barbera L and Neureiter D: Differentiation patterning of vascular smooth muscle cells (VSMC) in atherosclerosis. Virchows Arch 455: 171-185, 2009.

46. Saika S, Ikeda K, Yamanaka O, et al: Transient adenoviral gene transfer of Smad7 prevents injury-induced epithelial-mesenchymal transition of lens epithelium in mice. Lab Invest 84: 1259-1270, 2004

47. Tan X, Li Y and Liu Y: Paricalcitol attenuates renal interstitial fibrosis in obstructive nephropathy. J Am Soc Nephrol 17: 3382-3393, 2006.

48. Zeisberg M, Bottiglio C, Kumar N, Maeshima Y, Strutz F, Muller GA and Kalluri R: Bone morphogenic protein-7 inhibits progression of chronic renal fibrosis associated with two genetic mouse models. Am J Physiol Renal Physiol 285: F1060-F1067, 2003.

49. Jung A, Brabletz T and Kirchner T: The migrating cancer stem cells model - a conceptual explanation of malignant tumour progression. Ernst Schering Found Symp Proc: 109-124, 2006.

50. Handra-Luca A, Hong SM, Walter K, Wolfgang C, Hruban R and Goggins M: Tumour epithelial vimentin expression and outcome of pancreatic ductal adenocarcinomas. Br J Cancer 104 $1296-1302,2011$.

51. Hong SM, Li A, Olino K, et al: Loss of E-cadherin expression and outcome among patients with resectable pancreatic adenocarcinomas. Mod Pathol 24: 1237-1247, 2011

52. Jouppila-Matto A, Tuhkanen H, Soini Y, et al: Transcription factor snaill expression and poor survival in pharyngeal squamous cell carcinoma. Histol Histopathol 26: 443-449, 2011.

53. Franci C, Gallen M, Alameda F, Baro T, Iglesias M, Virtanen I and Garcia DH: Snaill protein in the stroma as a new putative prognosis marker for colon tumours. PLoS One 4: e5595, 2009.

54. Bieche I, Lerebours F, Tozlu S, Espie M, Marty M and Lidereau R: Molecular profiling of inflammatory breast cancer: identification of a poor-prognosis gene expression signature. Clin Cancer Res 10: 6789-6795, 2004.

55. Sarkar FH, Li Y, Wang Z and Kong D: Pancreatic cancer stem cells and EMT in drug resistance and metastasis. Minerva Chir 64: 489-500, 2009.

56. van Zijl F, Zulehner G, Petz M, et al: Epithelial-mesenchymal transition in hepatocellular carcinoma. Future Oncol 5: $1169-1179,2009$

57. Sabbah M, Emami S, Redeuilh G, et al: Molecular signature and therapeutic perspective of the epithelial-to-mesenchymal transitions in epithelial cancers. Drug Resist Updat 11: 123-151, 2008.

58. Thiery JP, Chua K, Sim WJ and Huang R: Epithelial mesenchymal transition during development in fibrosis and in the progression of carcinoma. Bull Cancer 97: 1285-1295, 2010 (In French).

59. Gupta PB, Onder TT, Jiang G, Tao K, Kuperwasser C, Weinberg RA and Lander ES: Identification of selective inhibitors of cancer stem cells by high-throughput screening. Cell 138: 645-659, 2009.
60. Ketola K, Hilvo M, Hyotylainen T, et al: Salinomycin inhibits prostate cancer growth and migration via induction of oxidative stress. Br J Cancer 106: 99-106, 2012.

61. Wang Y: Effects of salinomycin on cancer stem cell in human lung adenocarcinoma A549 cells. Med Chem 7: 106-111, 2011.

62. Gong C, Yao H, Liu Q, Chen J, Shi J, Su F and Song E: Markers of tumor-initiating cells predict chemoresistance in breast cancer. PLoS One 5: e15630, 2010.

63. Bardsley MR, Horvath VJ, Asuzu DT, et al: Kitlow stem cells cause resistance to Kit/platelet-derived growth factor alpha inhibitors in murine gastrointestinal stromal tumors. Gastroenterology 139: 942-952, 2010.

64. Fuchs D, Daniel V, Sadeghi M, Opelz G and Naujokat C: Salinomycin overcomes ABC transporter-mediated multidrug and apoptosis resistance in human leukemia stem cell-like KG-1a cells. Biochem Biophys Res Commun 394: 1098-1104, 2010.

65. Jones PA and Taylor SM: Cellular differentiation, cytidine analogs and DNA methylation. Cell 20: 85-93, 1980.

66. Jones PA, Taylor SM and Wilson V: DNA modification, differentiation, and transformation. J Exp Zool 228: 287-295, 1983.

67. Fraga MF, Herranz M, Espada J, et al: A mouse skin multistage carcinogenesis model reflects the aberrant DNA methylation patterns of human tumors. Cancer Res 64: 5527-5534, 2004

68. Herranz N, Pasini D, Diaz VM, et al: Polycomb complex 2 is required for E-cadherin repression by the Snaill transcription factor. Mol Cell Biol 28: 4772-4781, 2008.

69. Vincent A and Van SI: On the epigenetic origin of cancer stem cells. Biochim Biophys Acta 1826: 83-88, 2012.

70. Rodriguez-Paredes M and Esteller M: Cancer epigenetics reaches mainstream oncology. Nat Med 17: 330-339, 2011.

71. Ehrlich M: DNA hypomethylation in cancer cells. Epigenomics 1 239-259, 2009

72. Ehrlich M: DNA methylation in cancer: too much, but also too little. Oncogene 21: 5400-5413, 2002.

73. Fullgrabe J, Kavanagh E and Joseph B: Histone onco-modifications. Oncogene 30: 3391-3403, 2011.

74. Maeda G, Chiba T, Aoba T and Imai K: Epigenetic inactivation of E-cadherin by promoter hypermethylation in oral carcinoma cells. Odontology 95: 24-29, 2007.

75. Lombaerts M, van Wezel T, Philippo K, et al: E-cadherin transcriptional downregulation by promoter methylation but not mutation is related to epithelial-to-mesenchymal transition in breast cancer cell lines. Br J Cancer 94: 661-671, 2006.

76. Savagner P: The epithelial-mesenchymal transition (EMT) phenomenon. Ann Oncol 21 (Suppl 7): vii89-vii92, 2010.

77. Lin T, Ponn A, Hu X, Law BK and Lu J: Requirement of the histone demethylase LSD1 in Snail-mediated transcriptional repression during epithelial-mesenchymal transition. Oncogene 29: 4896-4904, 2010

78. Yang F, Sun L, Li Q, Han X, Lei L, Zhang H and Shang Y: SET8 promotes epithelial-mesenchymal transition and confers TWIST dual transcriptional activities. EMBO J 31: 110-123, 2011

79. Kalakonda N, Fischle W, Boccuni P, et al: Histone H4 lysine 20 monomethylation promotes transcriptional repression by L3MBTL1. Oncogene 27: 4293-4304, 2008.

80. Li Z, Nie F, Wang S and Li L: Histone H4 Lys 20 monomethylation by histone methylase SET 8 mediates Wnt target gene activation. Proc Natl Acad Sci USA 108: 3116-3123, 2011.

81. Bernstein BE, Mikkelsen TS, Xie X, et al: A bivalent chromatin structure marks key developmental genes in embryonic stem cells. Cell 125: 315-326, 2006.

82. Mongroo PS and Rustgi AK: The role of the miR-200 family in epithelial-mesenchymal transition. Cancer Biol Ther 10: 219-222, 2010.

83. Bullock MD, Sayan AE, Packham GK and Mirnezami AH: MicroRNAs: critical regulators of epithelial to mesenchymal (EMT) and mesenchymal to epithelial transition (MET) in cancer progression. Biol Cell 104: 3-12, 2012.

84. Vrba L, Jensen TJ, Garbe JC, et al: Role for DNA methylation in the regulation of miR-200c and miR-141 expression in normal and cancer cells. PLoS One 5: e8697, 2010.

85. Davalos V, Moutinho C, Villanueva A, Boque R, Silva P, Carneiro $\mathrm{F}$ and Esteller M: Dynamic epigenetic regulation of the microRNA-200 family mediates epithelial and mesenchymal transitions in human tumorigenesis. Oncogene 31: 2062-2074, 2012.

86. Wiklund ED, Bramsen JB, Hulf T, et al: Coordinated epigenetic repression of the miR-200 family and miR-205 in invasive bladder cancer. Int J Cancer 128: 1327-1334, 2011. 
87. Neves R, Scheel C, Weinhold S, et al: Role of DNA methylation in miR-200c/141 cluster silencing in invasive breast cancer cells. BMC Res Notes 3: 219, 2010.

88. Tryndyak VP, Beland FA and Pogribny IP: E-cadherin transcriptional down-regulation by epigenetic and microRNA-200 family alterations is related to mesenchymal and drug-resistant phenotypes in human breast cancer cells. Int J Cancer 126: 2575-2583, 2010.

89. Kelly TK, De Carvalho DD and Jones PA: Epigenetic modifications as therapeutic targets. Nat Biotechnol 28: 1069-1078, 2010.

90.Lane AA and Chabner BA: Histone deacetylase inhibitors in cancer therapy. J Clin Oncol 27: 5459-5468, 2009.

91. Stintzing S, Kemmerling R, Kiesslich T, Alinger B, Ocker M and Neureiter D: Myelodysplastic syndrome and histone deacetylase inhibitors: "to be or not to be acetylated"? J Biomed Biotechnol 2011: 214143, 2011.

92. Batty N, Malouf GG and Issa JP: Histone deacetylase inhibitors as anti-neoplastic agents. Cancer Lett 280: 192-200, 2009.

93. Ocker M: Deacetylase inhibitors - focus on non-histone targets and effects. World J Biol Chem 1: 55-61, 2010.

94. Ocker M and Schneider-Stock R: Histone deacetylase inhibitors: signalling towards p21cip1/waf1. Int J Biochem Cell Biol 39: 1367-1374, 2007.

95. Spange S, Wagner T, Heinzel T and Kramer OH: Acetylation of non-histone proteins modulates cellular signalling at multiple levels. Int J Biochem Cell Biol 41: 185-198, 2009.

96. Di Fazio P, Schneider-Stock R, Neureiter D, et al: The pan-deacetylase inhibitor panobinostat inhibits growth of hepatocellular carcinoma models by alternative pathways of apoptosis. Cell Oncol 32: 285-300, 2010.

97. Di Fazio P, Montalbano R, Quint K, et al: The pan-deacetylase inhibitor panobinostat modulates expression of epithelialmesenchymal transition markers in hepatocellular carcinoma models. Oncol Lett (In press).

98. Neureiter D, Zopf S, Leu T, et al: Apoptosis, proliferation and differentiation patterns are influenced by Zebularine and SAHA in pancreatic cancer models. Scand J Gastroenterol 42: 103-116, 2007.

99. Ryningen A, Stapnes C and Bruserud O: Clonogenic acute myelogenous leukemia cells are heterogeneous with regard to regulation of differentiation and effect of epigenetic pharmacological targeting. Leuk Res 31: 1303-1313, 2007.

100. Todaro M, Francipane MG, Medema JP and Stassi G: Colon cancer stem cells: promise of targeted therapy. Gastroenterology 138: 2151-2162, 2010

101.Ivanova T, Zouridis $\mathrm{H}$, Wu Y, et al: Integrated epigenomics identifies BMP4 as a modulator of cisplatin sensitivity in gastric cancer. Gut: Apr 25, 2012 (E-pub ahead of print).

102. Bhatla T, Wang J, Morrison DJ, Raetz EA, Burke MJ, Brown P and Carroll WL: Epigenetic reprogramming reverses the relapse-specific gene expression signature and restores chemosensitivity in childhood B-lymphoblastic leukemia. Blood 119: 5201-5210, 2012.

103. Omenetti A, Bass LM, Anders RA, et al: Hedgehog activity, epithelial-mesenchymal transitions, and biliary dysmorphogenesis in biliary atresia. Hepatology 53: 1246-1258, 2011.

104. Fabris L and Strazzabosco M: Epithelial-mesenchymal interactions in biliary diseases. Semin Liver Dis 31: 11-32, 2011.

105. Bailey JM, Singh PK and Hollingsworth MA: Cancer metastasis facilitated by developmental pathways: Sonic hedgehog, Notch, and bone morphogenic proteins. J Cell Biochem 102: 829-839, 2007.

106. Dasgupta P, Rizwani W, Pillai S, et al: Nicotine induces cell proliferation, invasion and epithelial-mesenchymal transition in a variety of human cancer cell lines. Int J Cancer 124: 36-45, 2009.

107.Li Y, Liu Y, Xu Y, Voorhees JJ and Fisher GJ: UV irradiation induces Snail expression by AP-1 dependent mechanism in human skin keratinocytes. J Dermatol Sci 60: 105-113, 2010.

108. Hardy KM, Booth BW, Hendrix MJ, Salomon DS and Strizzi L: ErbB/EGF signaling and EMT in mammary development and breast cancer. J Mammary Gland Biol Neoplasia 15: 191-199, 2010.
109. Guttilla IK, Adams BD and White BA: ERalpha, microRNAs, and the epithelial-mesenchymal transition in breast cancer. Trends Endocrinol Metab 23: 73-82, 2012.

110. Gallo D, Ferlini C and Scambia G: The epithelial-mesenchymal transition and the estrogen-signaling in ovarian cancer. Curr Drug Targets 11: 474-481, 2010.

111. Katoh Y and Katoh M: FGFR2-related pathogenesis and FGFR2-targeted therapeutics (Review). Int J Mol Med 23: 307-311, 2009.

112. Ding W, You H, Dang H, et al: Epithelial-to-mesenchymal transition of murine liver tumor cells promotes invasion. Hepatology 52: 945-953, 2010

113. Semenza GL: Hypoxia-inducible factors: mediators of cancer progression and targets for cancer therapy. Trends Pharmacol Sci 33: 207-214, 2012

114. Jiang J, Tang YL and Liang XH: EMT: a new vision of hypoxia promoting cancer progression. Cancer Biol Ther 11: 714-723, 2011.

115. Yang SY, Miah A, Pabari A and Winslet M: Growth Factors and their receptors in cancer metastases. Front Biosci 16: 531-538, 2011.

116. Mamuya FA and Duncan MK: aV integrins and TGF- $\beta$-induced EMT: a circle of regulation. J Cell Mol Med 16: 445-455, 2012.

117. Eble JA and Haier J: Integrins in cancer treatment. Curr Cancer Drug Targets 6: 89-105, 2006.

118. Lahsnig C, Mikula M, Petz M, et al: ILEI requires oncogenic Ras for the epithelial to mesenchymal transition of hepatocytes and liver carcinoma progression. Oncogene 28: 638-650, 2009.

119. Yadav A, Kumar B, Datta J, Teknos TN and Kumar P: IL-6 promotes head and neck tumor metastasis by inducing epithelialmesenchymal transition via the JAK-STAT3-SNAIL signaling pathway. Mol Cancer Res 9: 1658-1667, 2011.

120. Andrae J, Gallini R and Betsholtz C: Role of platelet-derived growth factors in physiology and medicine. Genes Dev 22: 1276-1312, 2008.

121. Peebles KA, Lee JM, Mao JT, et al: Inflammation and lung carcinogenesis: applying findings in prevention and treatment. Expert Rev Anticancer Ther 7: 1405-1421, 2007.

122. Lee JM, Yanagawa J, Peebles KA, Sharma S, Mao JT and Dubinett SM: Inflammation in lung carcinogenesis: new targets for lung cancer chemoprevention and treatment. Crit Rev Oncol Hematol 66: 208-217, 2008.

123. Gherardi E, Birchmeier W, Birchmeier C and Vande WG: Targeting MET in cancer: rationale and progress. Nat Rev Cancer 12: 89-103, 2012.

124. Tolnay E, Kuhnen C, Wiethege T, Konig JE, Voss B and Muller KM: Hepatocyte growth factor/scatter factor and its receptor c-Met are overexpressed and associated with an increased microvessel density in malignant pleural mesothelioma. J Cancer Res Clin Oncol 124: 291-296, 1998.

125. Heuberger $\mathrm{J}$ and Birchmeier W: Interplay of cadherin-mediated cell adhesion and canonical Wnt signaling. Cold Spring Harb Perspect Biol 2: a002915, 2010.

126. Hollier BG, Evans K and Mani SA: The epithelial-to-mesenchymal transition and cancer stem cells: a coalition against cancer therapies. J Mammary Gland Biol Neoplasia 14: 29-43, 2009.

127. Mimeault M and Batra SK: New advances on critical implications of tumor- and metastasis-initiating cells in cancer progression, treatment resistance and disease recurrence. Histol Histopathol 25: 1057-1073, 2010.

128. Singh A and Settleman J: EMT, cancer stem cells and drug resistance: an emerging axis of evil in the war on cancer. Oncogene 29: 4741-4751, 2010.

129. Wang Z, Li Y, Ahmad A, Azmi AS, Kong D, Banerjee S and Sarkar FH: Targeting miRNAs involved in cancer stem cell and EMT regulation: an emerging concept in overcoming drug resistance. Drug Resist Updat 13: 109-118, 2010.

130. Wellner U, Schubert J, Burk UC, et al: The EMT-activator ZEB1 promotes tumorigenicity by repressing stemness-inhibiting microRNAs. Nat Cell Biol 11: 1487-1495, 2009.

131. Wendt MK, Tian M and Schiemann WP: Deconstructing the mechanisms and consequences of TGF-beta-induced EMT during cancer progression. Cell Tissue Res 347: 85-101, 2012. 